CLINICAL REPORT

\author{
J.S. Hahn \\ P.D. Barnes \\ N.J. Clegg \\ E.E. Stashinko
}

\title{
Septopreoptic Holoprosencephaly: A Mild Subtype Associated with Midline Craniofacial Anomalies
}

\begin{abstract}
SUMMARY: HPE is a congenital brain malformation characterized by failure of the prosencephalon to divide into 2 hemispheres. We have identified 7 patients who have a mild subtype of HPE in which the midline fusion was restricted to the septal region or preoptic region of the telencephalon. This subtype, which we call septopreoptic HPE, falls in the spectrum of lobar HPE, but lacks significant frontal neocortical fusion seen in lobar HPE. Other imaging characteristics include thickened or dysplastic fornix, absent or hypoplastic anterior CC, and single unpaired ACA. The SP was fully formed in 4, partially formed in 2, and absent in 1 . Mild midline craniofacial malformation, such as $\mathrm{SMMCl}$ and CNPAS were found in $86 \%$ and $71 \%$, respectively. Patients outside of infancy often manifested language delay, learning disabilities, or behavioral disturbances, while motor function was relatively spared.
\end{abstract}

\begin{abstract}
ABBREVIATIONS: $\mathrm{AC}=$ anterior commissure; $\mathrm{ACA}=$ anterior cerebral artery; $\mathrm{CC}=$ corpus callosum; CNPAS = congenital nasal piriform aperture stenosis; FSPGR = fast-spoiled gradient-recalled $\mathrm{HPE}=$ holoprosencephaly; IHF = interhemispheric fissure; MPR = multiplanar reconstructed; $\mathrm{SMMCl}=$ single median maxillary central incisor; $\mathrm{SP}=$ septum pellucidum; $\mathrm{SPGR}=$ spoiled gradient-recalled; $\mathrm{V} 3$ = third ventricle
\end{abstract}

H $\mathrm{PE}$ is a complex congenital brain malformation characterized by failure of the forebrain to bifurcate into 2 hemispheres, a process normally complete by the fifth week of gestation. " As the name "HPE" implies, these nonseparated prosencephalic structures involve parts of the telencephalon and diencephalon. HPE has traditionally been classified according to DeMyer's 3 grades of severity: alobar, semilobar, and lobar. ${ }^{2}$ In addition to these classic forms, a subtype of HPE, the middle interhemispheric variant or syntelencephaly, has been characterized. ${ }^{3,4}$ In these types of HPE, there is significant nonseparation of the neocortex of the cerebral hemispheres. In the alobar type, the entire hemispheres are involved, and in the semilobar and lobar types, the frontal lobes are primarily involved. In the middle interhemispheric variant, there is nonseparation of the posterior frontal and anterior parietal lobes in the perirolandic region. We report a series of patients who have a very mild form of HPE, in which the nonseparation is restricted to the septal (subcallosal) and/or preoptic regions or both regions (Fig 1). Patients with this type of HPE often present initially with mild midline craniofacial malformations, including SMMCI and CNPAS. Our aim was to characterize the neuroimaging and clinical findings of a mild subtype of HPE that has less neocortical fusion than classic lobar HPE.

\section{Received January 14, 2010; accepted after revision March 15}

From the Departments of Neurology (J.S.H.) and Diagnostic Radiology (P.D.B.), Stanford University School of Medicine, Stanford, California; Texas Scottish Rite Hospital for Children (N.J.C.), Dallas, Texas; Kennedy Krieger Institute (E.E.S.), Baltimore, Maryland; and Lucile Packard Children's Hospital at Stanford (J.S.H., P.D.B.), Stanford, California.

This work was supported by the Carter Centers for Brain Research in Holoprosencephaly and Related Malformations, the Don and Linda Carter Foundation, and the Crowley-Carter Foundation.

Please address correspondence to Jin S. Hahn, MD, Department of Neurology, Stanford University Medical Center, 300 Pasteur Dr, Room A343, Stanford, CA 94305-5235; e-mail: jhahn@stanford.edu

DOI 10.3174/ajnr.A2123

\section{Materials and Methods}

We searched the neuroimaging data base of the Carter Centers for Research in Holoprosencephaly and Related Malformations for patients who met the criterion for a mild subtype that we call "septopreoptic HPE." The imaging criterion for inclusion was abnormal fusion restricted to the septal (subcallosal) and preoptic telencephalic regions (Fig 1). The exclusion criterion was more extensive fusion of the frontal lobes such as seen in classic lobar HPE. The search was restricted to patients who had previously been categorized as having lobar HPE, because patients with the semilobar, alobar, and middle interhemispheric variant would have been excluded because they have more extensive hemispheric fusion. The study was approved by the Stanford institutional review board.

The brain MR imaging studies were reviewed by a pediatric neuroradiologist (P.D.B.) by using a previously reported grading system for analyzing holoprosencephaly. ${ }^{4,5}$ Special attention was

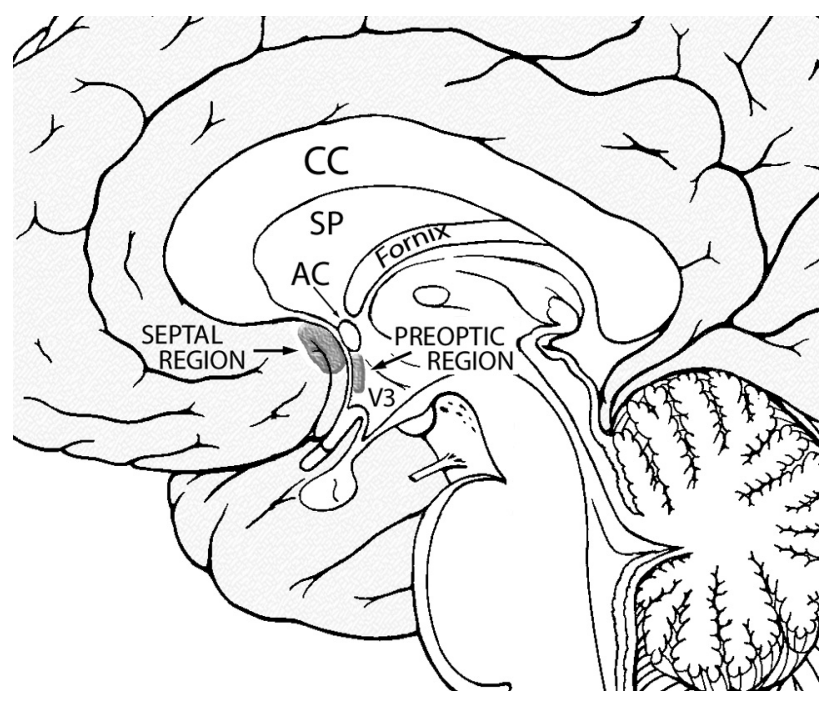

Fig 1. Diagram of the midsagittal view of the brain displaying the locations of the septal and preoptic regions. Modified from Martin J. ${ }^{22}$ 

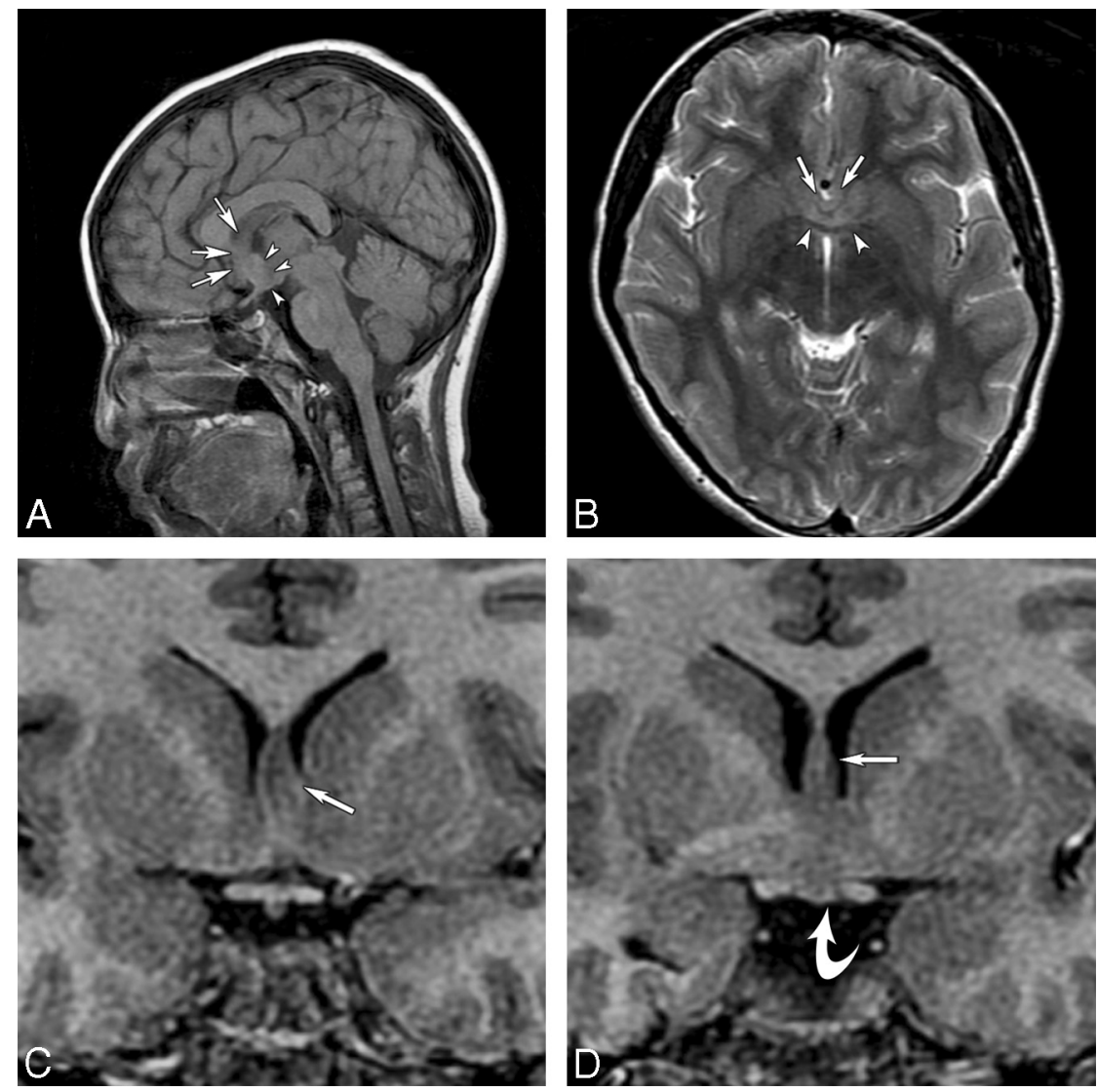

Fig 2. MR imaging of a 10-year-old boy with learning disabilities, a SMMCI, CNPAS, precocious puberty, and other endocrinopathies. $A$, T1-weighted midsagittal image shows hypoplasia of the rostrum of the CC and a rectangular area of abnormality in the subcallosal region, anterior to the hypothalamic region (arrowheads). The dysplastic-appearing fornix is anterior to this region (arrows). B, Axial T2-weighted image shows well-developed anterior and posterior interhemispheric fissures and an azygous ACA flow void in the anterior interhemispheric fissure. There is an area of midline fusion just anterior to the AC, which appears as a dark bowlike band (arrowheads). Further anterior to this region are the dysplastic fornix (arrows) and cortical gray matter that is continuous in the middle. C, Coronal SPGR image anterior to the AC shows an area of fusion of the septal region (arrow). $D$, Coronal SPGR image at the level to the AC shows dysplastic thickened fornices (arrow) traveling below the SP and inferiorly an area of midline fusion in the preoptic region and basal structures (curved arrow).

directed to the septal and preoptic regions for evidence of fusion or dysplasia. The studies were also evaluated for the presence of extracerebral malformations, specifically for the presence of SMMCI and CNPAS.

We reviewed the clinical information in the research data base for the patients who were evaluated by using the previously published methods at 1 of the Carter Centers. ${ }^{6}$ We queried for the presence of abnormalities of the neurologic examination, neurodevelopmental outcomes, and endocrinopathies.

\section{Results}

From a total of 434 patients with a confirmed diagnosis of HPE from neuroimaging studies, we identified 7 patients (1 male, 6 female) who met the criteria of septopreoptic HPE as defined above. The age range at the time of MR imaging was 4 days to 17 years. Figures 2-4 show 3 exemplary studies from patients with this disorder. All had fusion of the septal and preoptic regions. By definition, all patients had no or minimal fusion of the frontal neocortex. The anterior IHF and the anterior falx were present. Because the posterior hemispheres are not affected, the posterior IHF and falx were also well developed.

The CC was abnormally developed in all 7 patients. The mildest form of callosal abnormality was a hypoplastic rostrum (Fig 2A). Usually the rostrum was absent or hypoplastic, while the genu was less severely hypoplastic. The body and the splenium of the CC were normal in most patients, except in 1 in whom the entire CC was thin, especially anteriorly (Fig $3 A$ ).

The SP was fully formed in 4 (Fig 3C), partially formed in 2, and absent in 1 . The anterior portion of the fornix, a structure that is closely related to the SP, appeared thickened in 4 patients and dysplastic in 3 patients (Figs $2 A,-D$; $3 D$; and $4 C$, $-D)$. The AC was present in all of the non-neonatal cases. It was difficult to identify the $\mathrm{AC}$ in neonates due to lack of myelination.

The V3 was visible in all patients, though the anteriormost aspect of the V3 was often hypoplastic due to the fusion of the preoptic region. The lateral ventricles were formed and the frontal horns were present in all patients. However in those with a partial or absent SP, the frontal horns were small. Dorsal cyst and hydrocephalus were absent in all patients.

The caudate and lentiform nuclei appeared fully separated in all patients. In 2 of 7 patients, the thalamic nuclei appeared partially fused $(<50 \%)$ (Fig $4 C$ ), and in the remainder, they were normally separated. The pituitary gland appeared normal in all except 2 patients: 1 had a small anterior pituitary gland and another had a small posterior pituitary gland associated with an ectopic neurohypophysis (Fig 3A).

A single unpaired (azygous) ACA was found in the anterior interhemispheric fissure in all patients (Figs $2 B, 3 B$, and $4 B$ ). 

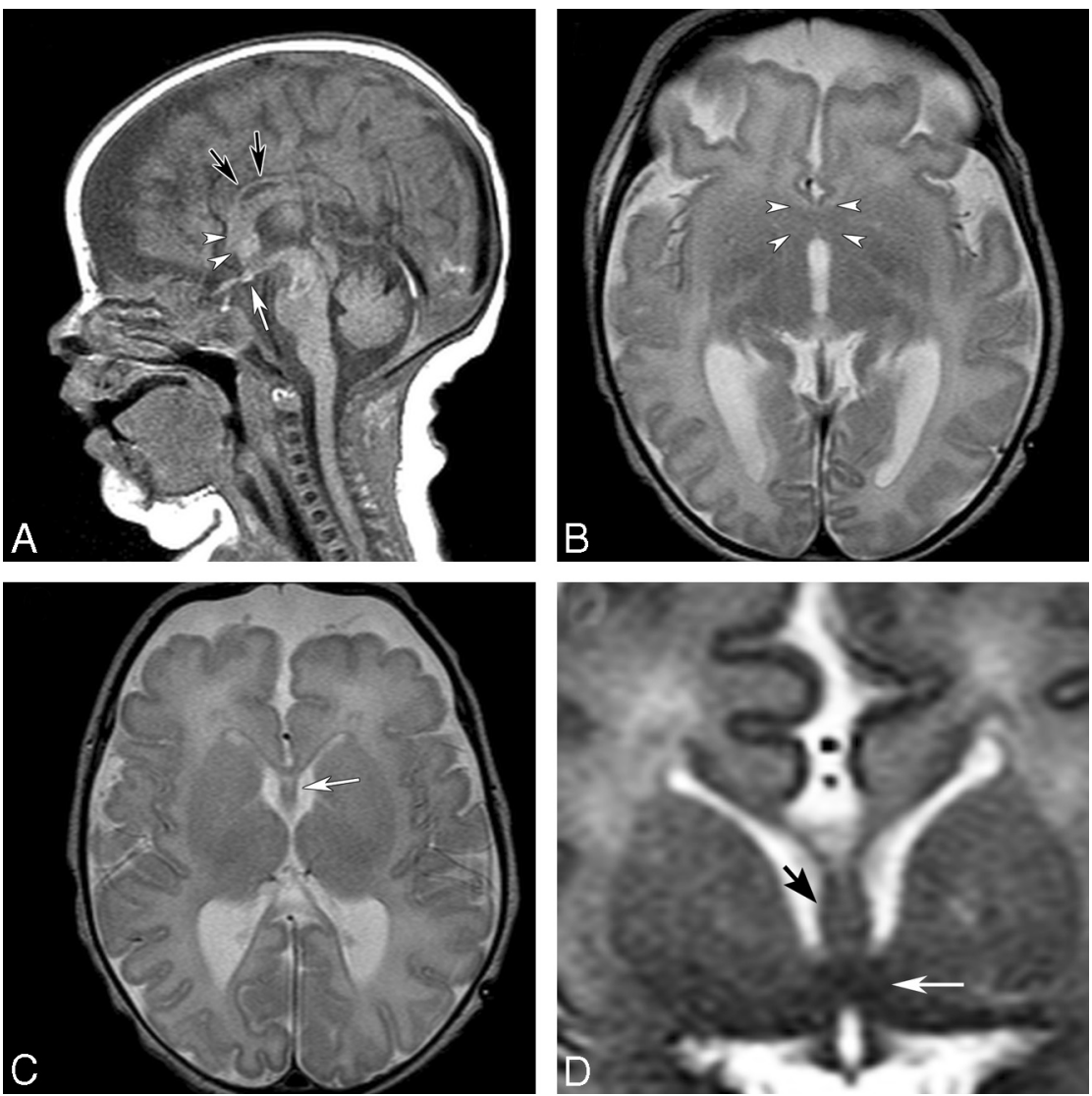

Fig 3. MR imaging of an 8-day-old term neonate with atrial and ventricular septal defects and SMMCl. A, Sagittal T1-weighted image shows a thin CC (black arrows), a thickened fornix and dysplastic subcallosal areas (arrowheads). A small ectopic pituitary gland is noted near the chiasm (white arrow). B, Axial T2-weighted image shows an area of fusion in the septal and preoptic regions (arrowheads). C, An azygous ACA is present in the anterior IHF. Axial T2-weighted image slightly superior shows the presence of the SP (white arrow) and a thin genu of the CC. $D$, Coronal T2-weighted image at the level of the AC shows fusion of the midline region (white arrow) below the fornices (black arrow)

Mild midline craniofacial malformations consisting of SMMCIs were identified in 6 of 7 patients $(86 \%$, not assessable in the imaging study of 1 patient), and CNPAS was found in 5 of 7 (71\%, not assessable in 2).

\section{Clinical Findings}

Four of the 7 patients were evaluated at 1 of the Carter Centers for Research in Holoprosencephaly and Related Brain Malformations. Case 1 (Fig 2) is a 10-year-old boy with multiple endocrinopathies (requiring thyroxine, cortisol, and desmopressin acetate [DDAVP] replacement), precocious puberty, attention deficit disorder, and impulse-control disorder. Neuropsychological testing at 10 years of age revealed that he was functioning at a 5- to 6-year level. An 8-year-old girl (case 6 in our series) had delayed expressive language development (began speaking at 3.5 years of age) and attention deficit disorder. Case 7, who was 20-years-old at follow-up, had mild learning disabilities but attended regular classes. She graduated from high school and also completed 1 year at a community college. These 3 patients were ambulatory and had no motor dysfunction.

Case 3 (Fig 4) is a complicated neonate with multiple congenital anomalies including coarctation of the aorta, vertebral and rib anomalies, and an anteriorly placed anus. She was hospitalized for 11 weeks and at discharge was noted to have generalized hypotonia and poor swallowing function requiring a gastrostomy tube for feedings. Her MR imaging also showed extensive white matter injury due to venous infarcts. In the 3 remaining patients, clinical data were not available because they had not been evaluated at 1 of the Carter Centers.

\section{Discussion}

Modern MR imaging has enabled detailed analysis of the cortical, white matter, and deep gray structural anomalies in HPE. This has allowed better classification of various types of HPE, identification of newer subtypes, and understanding of the pathogenesis. The sine qua non feature of HPE is an incomplete separation of the cerebral hemispheres, which results in a lack of cleavage (nonseparation) of midline telencephalic and diencephalic structures. Because the abnormally fused regions in septopreoptic HPE are telencephalic structures, this mild form meets the accepted criteria of HPE. ${ }^{7}$

However, classifying these mild midline abnormalities as HPE may be controversial. ${ }^{8}$ Many who study HPE consider the presence of the fornix, septum, and AC to essentially exclude the diagnosis of HPE (personal communication, A. James Barkovich, Jan. 5, 2005), especially when the 2 cerebral hemispheres appear nearly completely separated. Nevertheless, both the preoptic and septal regions are telencephalic structures (with the former being closely related in structure to the hypothalamus, a diencephalic structure). Therefore, by definition, a developmental nonseparation of telencephalic structures can be classified as HPE. ${ }^{7}$ The nonseparation of these midline structures would be consistent with the ventral 

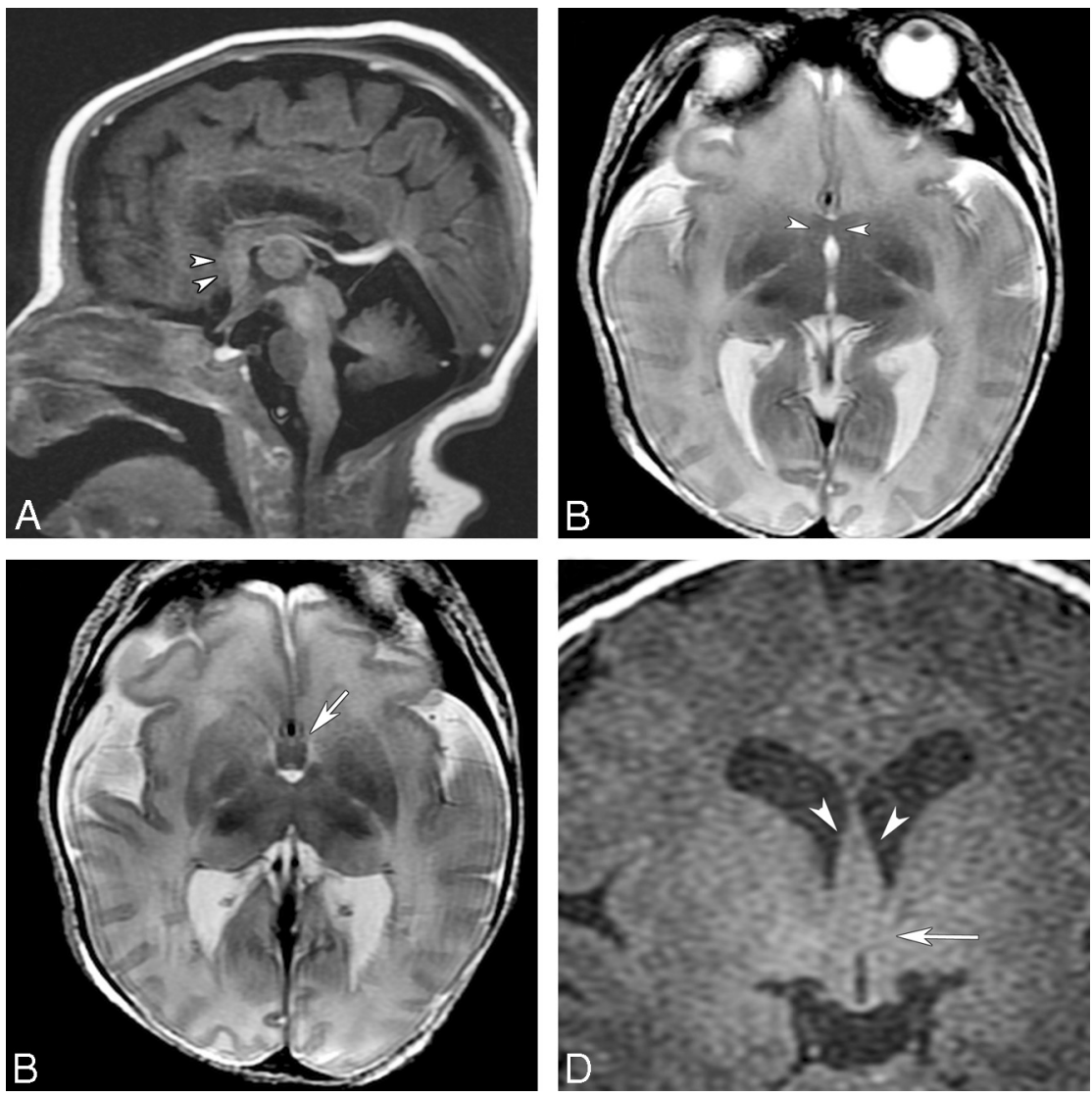

Fig 4. MR imaging of a 6-day-old term neonate initially diagnosed as having choanal atresia, vertebral anomalies, and coactation of aorta. $A$, Sagittal FSPGR image shows an area of fusion in the subcallosal region (arrowheads). B, Axial T2-weighted image shows the abnormal fusion in the same region (arrowheads). An azygous ACA is present in the anterior IHF. C, Axial T2-weighted image slightly superior shows thickened fornices (arrow) and partial fusion of the thalami. D, Coronal FSPGR image shows thickened fornices (arrowheads) and an area of fusion in the preoptic area (arrow).

\begin{tabular}{lll}
\hline \multicolumn{1}{l}{ Neuroimaging features comparing lobar versus septopreoptic HPE } & \\
\hline \multicolumn{1}{c}{ Lobar } & \multicolumn{1}{c}{ Septopreoptic } \\
\hline Cortical nonseparation & Basal frontal & Septal and preoptic regions \\
CC & Rostrum and genu absent; anterior body variably & $\begin{array}{c}\text { Rostrum absent or hypoplastic; genu hypoplastic; } \\
\text { body and splenium present }\end{array}$ \\
Anterior interhemispheric fissure and falx & present; splenium present & Fairly deep IHF \\
Ventricles & Rudimentary frontal horns; third ventricle formed & Normal or small frontal horns; third ventricle formed \\
Dorsal cyst & Absent & Absent \\
SP & Absent & Present or dysplastic, rarely absent \\
Hypothalamus & Often fused to some degree (83\%) & Anterior hypothalamus often fused \\
Cerebral vasculature & Azygous anterior cerebral artery (more anteriorly & Azygous anterior cerebral artery (more posteriorly \\
& displaced) & placed) \\
\hline
\end{tabular}

patterning defect seen in classic HPE, though the affected midline region is much more restricted. We believe that the condition in these patients represents a milder form of lobar HPE, which is at the mildest end of the spectrum of HPE. These patients do not merely have a "microform of HPE," which is defined as midline craniofacial anomaly in the absence of brain malformation.

Septopreoptic HPE is commonly included in the lobar spectrum, though distinct differences exist as outlined in the Table. In both subtypes, the frontal and occipital lobes are separated at least to some degree. However, in lobar HPE, there is fusion of the rostral/ventral aspects of the frontal lobes, whereas in septopreoptic HPE, there is no significant fusion of these regions. The anterior IHF and falx cerebri are present in both subtypes, though in lobar HPE, they are hypoplastic (ie, shallower than in septopreoptic HPE) due to the frontal lobe fusion. ${ }^{7}$

Similar to the finding of azygous ACA in lobar $\mathrm{HPE}^{7}$ we found that an unpaired ACA was also present in septopreoptic HPE. However, in lobar HPE, the azygous ACA is located more anteriorly (rostrally) due to the shallow anterior IHF. In contrast, in septopreoptic HPE, the azygous ACA is located posteriorly, near the subcallosal region.

The septal region, a telencephalic structure, includes both cortical and subcortical areas that belong to the forebrain (Fig 1). ${ }^{9}$ It forms part of the medial wall of the cerebral hemispheres. The cortical areas, called the septal cortex, are found under the rostrum of the CC (subcallosal area). Nuclei that lie deep to this region are called the septal nuclei. The subcallosal area is bordered dorsally by the CC and caudally by the AC 
and the preoptic region (Fig 1). ${ }^{10}$ Functionally, the septal region includes major hypothalamic and limbic structures that are interconnected via the hippocampal formation. Lesions in the septal region have been known to be associated with behavioral over-reaction to external stimuli. ${ }^{10}$

The preoptic region is a narrow vertical strip flanking the rostralmost part of the V3 that extends from the AC to the rostral part of the optic chiasm (Fig 1). ${ }^{11}$ Although most of the preoptic region is caudal to the lamina terminalis, a small portion is located rostrally. ${ }^{12}$ The preoptic region is thought to be telencephalic in origin, though later studies show a diencephalic origin. ${ }^{12}$ Regardless of its embryonic origin, it is closely related structurally and functionally to the hypothalamus. It contains 3 rather poorly differentiated cell masses: the preoptic periventricular nucleus, medial preoptic nucleus, and lateral preoptic nucleus. This region is implicated in regulating blood volume and pressure.

The primary defect in classic HPE is in the abnormal patterning signals from the rostral neural tube that occur during the first 4 weeks of gestation. ${ }^{1}$ In normal embryonic forebrain development, a complex interaction between ventral patterning signals (primarily sonic hedgehog from the floor plate and prechordal plates) and dorsal patterning signals (molecules arising from the roof plate) modulate regional identity of tissues along the vertical axis of the neural tube. ${ }^{7}$ The gradients of ventral and dorsal signaling molecules induce a distinct combination of genes and transcription factors in populations of cells at different levels along the vertical axis. The presumed mechanism in HPE is thought to be the lack of production of ventralizing factors or an overproduction of dorsalizing factors resulting in noncleavage of the midline structures, and producing holoprosencephalic brains. ${ }^{1}$ In septopreoptic HPE, the area of abnormal patterning seems to be restricted to a small forebrain region. As in classic HPE, the same signaling abnormalities can result in midline craniofacial malformations, albeit mild in phenotype.

The frequent association of anomalies of the fornix, SP, and anterior CC in septopreoptic HPE is most likely related to the embryonic relationship of these structures during weeks 6-8 of development. These structures all originate from the lamina reuniens. The lamina reuniens is located in the midline ventral region at the base of the interhemispheric cleft, dorsal to the lamina terminalis. ${ }^{13}$ The lamina reuniens will further develop into 2 distinct areas. The ventral part becomes the area praecommissuralis, which will develop into the septal region and AC. The dorsal part of the lamina reuniens becomes the hippocampal primordium, which will give rise to the fornix, hippocampal commissure, CC, and the cavum SP. It is likely that a disturbance in the development of the lamina reuniens leads to the structural abnormalities seen in septopreoptic HPE.

Patients with septopreoptic HPE are often referred to neuroimaging because of their mild midline malformations such as SMMCI and CNPAS. These midline malformations are also commonly seen in classic HPE. The otolaryngology literature is filled with examples of patients with mild HPE who first presented for nasal airway problems. ${ }^{14-16}$ CNPAS has also been associated with a high incidence of hypothalamicpituitary-adrenal axis dysfunction but is not necessarily associated with HPE. ${ }^{17}$ The presence of SMMCI may also be a clue to HPE. ${ }^{18-21}$ When these extracerebral anomalies are found either clinically or on imaging studies, one should perform high-resolution MR imaging. If an obvious diagnosis of classic HPE is not readily evident, careful attention should be directed to the preoptic area and septal region to look for the milder forms of HPE.

Even with the restricted area of midline fusion, patients with septopreoptic HPE also have neurodevelopmental and endocrine abnormalities. Unlike classic HPE, motor function appears to be spared. This is likely due to the lack of hemispheric and basal ganglia fusion that is common in classic HPE. The 1 exception in our series was a neonate (case 3 ) who had multiple congenital anomalies and white matter injury that most likely had a significant effect on her developmental outcome. More detailed clinical studies are needed to more accurately determine the neurodevelopmental outcome in septopreoptic HPE.

\section{Conclusions}

Septopreoptic HPE is a mild subtype of a midline developmental malformation characterized by restricted areas of midline fusion involving the septal and preoptic regions. It is commonly included in the lobar spectrum of HPE but appears to represent a milder form because there is a lack of significant neocortical fusion of the frontal lobes. It is often associated with neurodevelopmental problems and specific midline craniofacial malformations (SMMCI and CNPAS), which often may be the presenting finding.

\section{Acknowledgment}

We thank James Barkovich, MD, for his contribution to the neuroimaging concepts in HPE.

\section{References}

1. Golden JA. Towards a greater understanding of the pathogenesis of holoprosencephaly. Brain Dev 1999;21:513-21

2. DeMyer W. Holoprosencephaly (cyclopia-arrhinencephaly). In: Vinken PJ, Bruyn GW, Klawans HL, eds. Handbook of Clinical Neurology. 6th ed. Amsterdam, the Netherlands: Elsevier Science Publishers; 1987:225-44

3. Lewis AJ, Simon EM, Barkovich AJ, et al. Middle interhemispheric variant of holoprosencephaly: a distinct cliniconeuroradiologic subtype. Neurology 2002;59:1860-65

4. Simon EM, Hevner RF, Pinter JD, et al. The middle interhemispheric variant of holoprosencephaly. AJNR Am J Neuroradiol 2002;23:151-55

5. Simon EM, Hevner R, Pinter JD, et al. Assessment of the deep gray nuclei in holoprosencephaly. AJNR Am J Neuroradiol 2000;21:1955-61

6. Plawner LL, Delgado MR, Miller VS, et al. Neuroanatomy of holoprosencephaly as predictor of function: beyond the face predicting the brain. Neurology 2002;59:1058-66

7. Simon EM, Barkovich AJ. Holoprosencephaly: new concepts. Magn Reson Imaging Clin N Am 2001;9:149-64

8. Hahn JS, Barnes PD. Neuroimaging advances in holoprosencephaly: refining the spectrum of the midline malformation. Am J Med Genet C Semin Med Genet 2010;154C:120-32

9. Hendelman W. The limbic system. In: Atlas of Functional Neuroanatomy. 2nd ed. Boca Raton, Florida: Taylor \& Francis Group; 2006:202-38

10. Tamraz JC, Comair YG. The basal forebrain, diencephalon and basal ganglia. In: Atlas of Regional Anatomy of the Brain Using MRI. Berlin, Germany: SpringerVerlag; 2006:185-225

11. Nieuwenhuys R, Voogd J, van Huizen C. Olfactory and limbic system. In: The Human Central Nervous System: A Synopsis and Atlas. 2nd ed. New York: Springer-Verlag; 1981:185-215

12. Martin J. Hypothalamus. In: Neuroanatomy: Text and Atlas. New York: Elsevier Science Publishing Co; 1989:350-73

13. Griffiths PD, Batty R, Reeves MJ, et al. Imaging the corpus callosum, septum pellucidum and fornix in children: normal anatomy and variations of normality. Neuroradiology 2009;51:337-45 
14. Tavin E, Stecker E, Marion R. Nasal pyriform aperture stenosis and the holoprosencephaly spectrum. Int J Pediatr Otorhinolaryngol 1994;28:199-204

15. Chan EY, Ng DK, Chong AS, et al. Congenital nasal pyriform aperture stenosis with semilobar holoprosencephaly. Int J Pediatr Otorhinolaryngol 2005;69:93-96

16. Hui Y, Friedberg J, Crysdale WS. Congenital nasal pyriform aperture stenosis as a presenting feature of holoprosencephaly. Int J Pediatr Otorhinolaryngol 1995;31:263-74

17. Guilmin-Crepon S, Garel C, Baumann C, et al. High proportion of pituitary abnormalities and other congenital defects in children with congenital nasal pyriform aperture stenosis. Pediatr Res 2006;60:478-84

18. El-Jaick KB, Fonseca RF, Moreira MA, et al. Single median maxillary central incisor: new data and mutation review. Birth Defects Res A Clin Mol Teratol 2007;79:573-80

19. Berry SA, Pierpont ME, Gorlin RJ. Single central incisor in familial holoprosencephaly. J Pediatr 1984;104:877-80

20. Garavelli L, Zanacca C, Caselli G, et al. Solitary median maxillary central incisor syndrome: clinical case with a novel mutation of sonic hedgehog. $\mathrm{Am}$ Med Genet A 2004;127:93-95

21. Hattori H, Okuno T, Momoi T, et al. Single central maxillary incisor and holoprosencephaly. Am J Med Genet 1987;28:483-87

22. Martin J. Hypothalamus. In: Neuroanatomy: Text and Atlas. New York: Elsevier Science Publishing; 1989:350-73 